
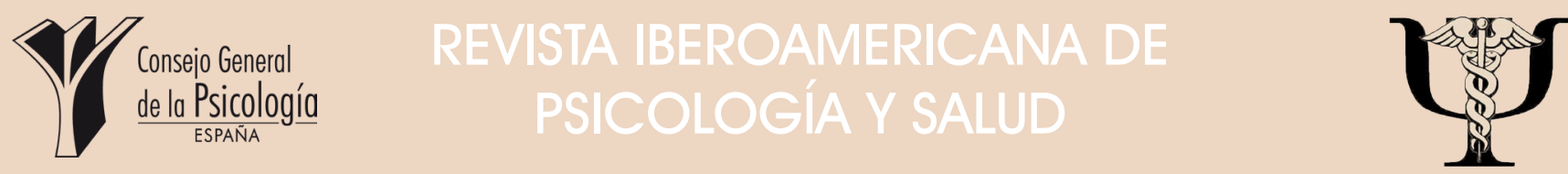

Revista Oficial de la Federación Iberoamericana de Asociaciones de Psicología (FIAP)

[Official Journal of the Latin-American Federation of Psychological Associations]

\title{
Factores psicosociales del consumo de verduras en niños escolarizados mexicanos de poblaciones urbanas y semi-urbanas
}

\section{Gerardo Ochoa-Meza ${ }^{1 *}$, Juan Carlos Sierra², Carmen Pérez-Rodrigo3, Javier Aranceta- Bartrina $^{4}$, Óscar A. Esparza-Del Villar ${ }^{1}$ y Bertha Musi-Lechuga'}

'Departamento de Ciencias Sociales, Universidad Autónoma de Ciudad Juárez (México)

${ }^{2}$ Centro de Investigación Mente, Cerebro y Comportamiento (CIMCYC), Universidad de Granada (España).

3Unidad de Nutrición Comunitaria, Bilbao (España).

${ }^{4}$ Departamento de Ciencias de la Alimentación, Fisiología y Toxicología, Universidad de Navarra (España).

- Recibido: 14 - Enero - 2017 . Aceptado: 20 - Abril - 2017 . Avance online: 12 - Mayo - 2017

RESUMEN. El objetivo del presente estudio es explorar el poder predictivo de factores psicosociales de motivación, habilidad y oportunidad ambiental asociados al consumo de verduras, en niños escolarizados de 10 a 12 años que residen en poblaciones urbanas y semi-urbanas de seis ciudades del Estado de Chihuahua (México). Se aplicó un cuestionario validado en una muestra representativa de 1.434 niños de Educación Primaria. La frecuencia de consumo diario fue mayor en niños semi-urbanos (verduras $\geq 2$ porciones/al día). Los factores en los análisis de regresión explicaron en población urbana y semi-urbana del 33\% $(p<0.01)$ al 45\% $(p<0.01)$ de la varianza en el consumo de verdura. Las preferencias, habilidades cognitivas, percepción de barreras y accesibilidad en la casa se asociaron al consumo de verduras en niños urbanos $(B<0.18, p<0.01)$ y semi-urbanos $(B<0.11, p<0.01)$. Se concluye que el diseño de intervenciones para fomentar el consumo deberá centrarse en incrementar las preferencias y habilidades cognitivas, en disminuir la percepción de barreras y facilitar la accesibilidad en la casa. Finalmente, se discuten las implicaciones de los hallazgos sobre los factores psicosociales y la necesidad de realizar análisis de mediación en futuras investigaciones para determinar su capacidad y fuerza predictiva para el logro de intervenciones efectivas. PALABRAS CLAVE: Factores psicosociales, Consumo de verduras, Cuestionario, Niños, México.

Psychosocial factors of vegetable intake in urban and semi-urban Mexican schoolchildren.

ABSTRACT. The aim of this study is to explore a predictive capacity of psychosocial factors of motivation, ability and environmental opportunity factors of vegetable intake between urban and semi-urban schoolchildren population 10 to 12 years old of six cities of the State of Chihuahua (Mexico). A validated questionnaire was administered in a representative sample of 1434 schoolchildren of Elementary School. Frequency of daily consumption of vegetables was higher in semi-urban schoolchildren (vegetables $\geq 2$ portion/day). The regression analysis explained 33\% ( $p<.01$ ) to $45 \%(p<.01)$ of the variance in vegetable intake by potential psychosocial factors. Preferences, cognitive abilities, perceived barriers and accessibility at home were a significant related to vegetable intake in urban $(B<.18, p<.01)$ and semi-urban schoolchildren $(B<.11, p<.01)$. It is concluded that in designing effective intervention to increment vegetable intake between urban and semi-urban children should focus on increase the preferences and cognitive abilities. Also, decrease perceived barriers and facilitate the availability at home. Finally, implication of the findings in psychosocial factors and for more efficient future research interventions need to be applied on mediation analysis to determine the predictive capacity and strength of the factors to obtain more effective intervention.

KEYWORDS: Psychosocial factors, Vegetable intake, Questionnaire, Children, Mexico.

\footnotetext{
*Correspondencia: Gerardo Ochoa-Meza.

Departamento de Ciencias Sociales, Universidad Autónoma de Ciudad Juárez.

Código Postal: 32310, Juárez, Chihuahua (México).

E-mail:gochoaster@gmail.com
}

(C) 2017 Sociedad Universitaria de Investigación en Psicología y Salud. Publicado por Consejo General de Colegios Oficiales de Psicólogos, España. Este es un artículo Open Access
bajo la CC BY-NC-ND licencia (http://creativecommons.org/licencias/by-nc-nd/4.0/).

Citar como/Cite as: Ochoa, G., Sierra, C. J., Pérez. C., Aranceta. J., Esparza. O. A., \& Musi. B. (2017). Factores psicosociales del consumo de verduras en niños escolarizados mexicanos de poblaciones urbanas y semi-urbanas. Revista Iberoamericana de Psicología y Salud, 8(2), 108-120, https://doi.org/10.23923/i.rips.2017.08.010
El bajo consumo de frutas y verduras ( $C$ F/V) se ha asociado con el desarrollo de algunas enfermedades crónicas como ciertos tipos de cáncer, algunas alteraciones cardiovasculares, accidentes cerebrovasculares, diabetes tipo 2 y obesidad (Boeing et al., 2012). Sin embargo, 
la epidemia global de la obesidad infantil y sus repercusiones sanitarias en la edad adulta han fomentado el incremento en el C FN (World Health Organization, WHO, 2016). Algunas evidencias epidemiológicas sugieren que un alto C F/V puede proteger en contra del desarrollo de enfermedades crónicas y ser una estrategia efectiva en la prevención de la obesidad en niños de edad escolar (Boeing et al., 2012; Diep, Chen, Davies, Baranowski y Baranowski, 2014; Klepp et al., 2005). Por tanto, la escuela es un componente clave en la prevención de dichas enfermedades y de la obesidad infantil. Además, la escuela es un escenario idóneo para la aplicación de intervenciones dirigidas a la modificación del comportamiento dietético del C F/V en niños y adolescentes (Baranowski, Diepp y Baranowski, 2013; Krølner et al., 2012).

Algunos estudios de revisión sobre programas de intervención han puesto de manifiesto la necesidad de identificar a los predictores del C F/V y de analizar sus diferencias en los niños y adolescentes (Guillaumie, Godin y Vézina-Im 2010; Krølner et al., 2011; Mclain, Chapuis, Nguyen-Rodríguez, Yaroch y Spruij-Metz, 2009; Rasmussen et al., 2006). De esta forma, en el abordaje de los problemas del bajo CF/ es crucial conocer la capacidad mediadora de los factores psicosociales en la predicción del comportamiento dietético (Krølner et al., 2012). Además, resulta necesario emplear instrumentos válidos y fiables que evalúen dichos factores (Agudo, 2005; De Bourdeaudhuij et al., 2005; Wolf et al., 2005; Yngve et al., 2005). Todo ello, centrado en la aplicación de estrategias educativas aplicables en niños escolarizados (Pérez-Rodrigo et al., 2005; Townsen y Foster, 2011).

El estudio de las variables mediadoras (como las preferencias, la accesibilidad en la escuela y en la casa) y su interacción con las variables moderadoras (por ejemplo, el sexo, el nivel socioeconómico o la influencia de la urbanización en el C F/N) facilita la comprensión de los enlaces entre dichas variables incrementando su efectividad en las intervenciones (Baranowski, 2011 ) como en la explicación del C F/N (Guillaumie et al., 2010; Krølner et al., 2011; Rasmussen et al., 2006) y en el desarrollo de modelos teóricos y predictivos (Brug, de Vel, de Nooijer y Verplanken, 2006; Hardeman et al., 2005; McClain et al., 2009).

El presente estudio se sustenta en el modelo socio-ecológico de conducta saludable (Sallis,
Owen y Fisher, 2008), el cual explica la conducta alimentaria saludable desde factores individuales y socio-ambientales (Brug, Kremers, Lenthe, Ball y Crawford, 2008; Graham, Pelletier, NeumarkSztainer, Lust y Laska, 2013; Vanwolleghem et al., 2016). Este modelo ha mostrado validez y capacidad predictiva y es uno de los más empleados para el estudio del C FN, la obesidad, la actividad física y el peso corporal (Blanchard et al., 2005; Cardon et al., 2012; D'Haese et al., 2015; Gentile et al., 2009; Vanwolleghem et al., 2016). Dicho modelo, incluye predictores derivados de la teoría de la influencia triádica (Flay y Petraitis, 1994) y se apoya en el análisis e intervenciones de multinivel (Moore, de SilvaSanigorski y Moore, 2013; Robinson, 2008), que se caracteriza por la manera de analizar las múltiples influencias de los factores individuales y socio-ambientales sobre la modificación de la conducta saludable (Sallis et al., 2008).

Existen pocos estudios sobre la influencia de la urbanización en el C FN (Rasmussen et al., 2006). Por ello, en el presente trabajo se explora la capacidad predictiva de los factores asociados con el consumo de verduras $(C N)$, con énfasis en las influencias individuales y socio-ambientales sobre el tipo de población (urbana y semi-urbana) en la que residen los niños escolarizados desde la perspectiva del modelo socio-ecológico de conducta saludable del C/V (Brug, Tak, Velde, Bere y De Bourdeaudhuii, 2008; Rothschild, 1999; Sallis et al., 2008).

Por lo tanto, el objetivo del presente estudio es explorar el poder predictivo de factores psicosociales de motivación, habilidad y oportunidad ambiental asociados con el consumo de verdura en niños escolarizados que residen en poblaciones urbanas y semi-urbanas, de seis ciudades del Estado de Chihuahua, México. Se establece la hipótesis de que los niños escolarizados que residen en poblaciones semiurbanas muestran un menor gusto y preferencias por las verduras, menos habilidades cognitivas para el consumo diario, perciben mayores obstáculos y tienen menos accesibilidad a las verduras para su consumo.

\section{MÉTODO}

\section{- PARTICIPANTES Y DISEÑO}

Se realizó un estudio transversal en el que 
participaron 1.434 niños seleccionados a través de un muestreo probabilístico, con submuestreo multietápico. El esquema de selección se efectuó por grado escolar, sexo y tipo de ciudad (urbana y semi-urbana) en la que habitualmente residen los niños escolarizados en diferentes centros de Educación Primaria del Estado de Chihuahua (México). De acuerdo con la distribución por grado escolar fueron 775 niños de $5^{\circ}$ y 659 de $6^{\circ}$ grado de un total de 41 grupos escolares de 16 escuelas, por sexo fueron 710 niñas y 724 niños, y, por ciudad, fueron 800 niños urbanos y 634 niños semi-urbanos; se incluyeron dos ciudades clasificadas como urbanas (Juárez y Chihuahua) por el número de habitantes, de 800.000 a 1.500.000, y cuatro semi-urbanas (Cuauhtémoc, Delicias, Hidalgo del Parral y Nuevo Casas Grandes, de 59.000 a 155.000) definidas por el Instituto Nacional de Estadística y Geografía (2010).

La fórmula para determinar el tamaño de la muestra fue: $n=\frac{Z^{2} p q N}{(N-1) e^{2}+Z^{2} p q}$ y se calculó al $3 \%$ de margen de error con un nivel de confianza del 95\% de acuerdo a la población total de alumnos $(N=106.195)$ de $5^{\circ}$ (53.193 alumnos) y $6^{\circ}$ (53.002 alumnos) grado de Educación Primaria de seis ciudades del Estado de Chihuahua (Servicios Educativos del Estado de Chihuahua, 2014). El tamaño de la muestra resultante fue de 1.057 participantes. Sin embargo, cuando se aplicaron los cuestionarios a los alumnos seleccionados por el esquema de muestreo, se obtuvieron 377 cuestionarios más, debido a su disponibilidad en el momento de su aplicación, que se tabularon según una selección aleatoria de la submuestra a la que correspondía, tratando de mejorar la significación de los parámetros establecidos para el cálculo de la misma. De esta forma, el tamaño de la muestra resultante fue de 1.434 participantes.

\section{- INSTRUMENTO}

Se utilizó la versión mexicana validada del cuestionario Pro Children Project Questionnaire (PCHPQ) que evalúa factores psicosociales asociados al consumo de verdura (OchoaMeza, Sierra, Pérez-Rodrigo y Aranceta-Bartrina, 2014; Ochoa-Meza, Sierra, Pérez-Rodrigo, Aranceta Bartrina y Esparza-Del Villar, 2014). El PCHPQ evalúa seis factores vinculados al CN (preferencias, habilidades cognitivas, percepción de barreras, modelación, accesibilidad en la casa y accesibilidad en la escuela) clasificados de acuerdo a lo propuesto por Brug, et al. (2008), Rothschild (1999) y Sallis et al. (2008) en las siguientes categorías: motivación (preferencias), habilidad (habilidades cognitivas y percepción de barreras) y oportunidad ambiental (modelación, accesibilidad en la casa y accesibilidad en la escuela). Así, por ejemplo, las preferencias fueron consideradas como parte de la dimensión de la motivación - subconstructo de la misma. Las preferencias fueron medidas con una lista de 11 verduras en una escala Likert de cinco opciones de respuesta que, oscilaron desde "me gusta mucho" hasta "no la he probado". Las habilidades cognitivas fueron medidas con 10 ítems de cinco categorías de respuesta cada uno, que van desde "No estoy totalmente de acuerdo" a "Estoy totalmente de acuerdo". La estimación de la percepción de barreras se evaluó mediante tres ítems con la misma escala Likert empleada en las habilidades cognitivas. La modelación, la accesibilidad en la casa y accesibilidad en la escuela también se estimaron con una escala Likert de "Nunca" a "Si, siempre". Finalmente, la medición de la frecuencia de consumo se realizó con un ítem de ocho categorías de respuesta en un rango de "Nunca" a "Todos los días, más de dos veces al día" validado dentro de la versión mexicana del cuestionario $P C H P Q$. Las características psicométricas del PCHPQ mostraron una moderada fiabilidad testretest (coeficiente de correlación intraclase $(\mathrm{CCl}$ ) $>0.60$ ) en todos los constructos de verdura en un rango de 0.60 a 0.68 . La consistencia interna de las escalas fue de moderada a alta $(\alpha=0.72$ 0.92) y la validez predictiva fue moderada a buena en un rango de correlaciones de Spearman de 0.40 a 0.60 en factores personales o individuales y de 0.14 a 0.40 en factores ambientales (OchoaMeza, Sierra, Pérez-Rodrigo, Aranceta Bartrina y Esparza-Del Villar, 2014).

\section{- PROCEDIMIENTO Y ANÁLISIS ESTADÍSTICOS}

Una vez que las autoridades escolares aprobaron la realización del estudio en los centros escolares se aplicó un cuestionario autoadministrado (Ochoa-Meza, Sierra, PérezRodrigo y Aranceta-Bartrina, 2014; OchoaMeza, Sierra, Pérez-Rodrigo, Aranceta Bartrina y Esparza-Del Villar, 2014) durante las horas de clase. Se informó a los participantes en el estudio 
que sus respuestas serían totalmente anónimas y confidenciales. Se utilizó el consentimiento tácito de los padres respecto a la participación de sus hijos, además se solicitó el consentimiento de los niños al iniciar la aplicación del instrumento.

Se utilizó el análisis multivariado de varianza (MANOVA) para examinar las diferencias entre las medias de los factores psicosociales de motivación, habilidad y oportunidad ambiental asociados al $\mathrm{C} / \mathrm{V}$ entre los niños que residen en poblaciones urbanas y semi-urbanas. Adicionalmente, para comparar las medias de los factores se aplicó la prueba $t$ de muestras independientes con un valor de significación estadística de $(p<0.05$.) Además, se utilizó la $d$ de Cohen (1992) para establecer el tamaño de la diferencia entre las medias.

Por otra parte, se estimaron las correlaciones de Pearson entre el CN y los predictores para el grupo de niños urbanos y semi-urbanos. Posteriormente, mediante la prueba $Z$ se compararon las correlaciones entre ambos grupos. Las correlaciones que resultaron mayores a 0.30 fueron incluidas en el modelo de análisis de regresión lineal múltiple con un valor estadísticamente significativo $(p<0.01)$, con el fin de reducir la multicolinealidad.

Se calculó el análisis de regresión lineal múltiple en cada uno de los grupos de niños que residen en poblaciones urbanas y semiurbanas. Se emplearon los coeficientes beta estandarizados y las correlaciones semiparciales para comparar los predictores y los modelos de regresión resultantes. Los datos fueron analizados con el programa estadístico para las ciencias sociales versión 20 (SPSS INC., 2011).

\section{RESULTADOS}

\section{- ESTADÍSTICA DESCRIPTIVA}

La Tabla 1 presenta la distribución de la frecuencia de CN en las muestras de niños escolarizados que residen en poblaciones urbanas y semi-urbanas. Ambas muestras presentaron pocas diferencias en los porcentajes de CN . El 24\% los niños urbanos y semi-urbanos reportaron un consumo diario de una, dos y más de dos veces al día, pero una mayor proporción de niños semiurbanos consumen verduras dos veces al día (6.6\%) o más de dos veces al día (6.0\%) que la proporción de niños urbanos que consume dos veces al día (4.3\%) y más de dos veces al día (5.4\%).

\section{- DIFERENCIAS EN LOS FACTORES DEL CONSUMO DE VERDURA ENTRE NIÑOS URBANOS Y SEMI- URBANOS}

El análisis del MANOVA de los factores psicosociales como variables dependientes fue significativo $(F(6)=2.03, p<0.05)$. Las medias de los factores analizados se muestran en la Tabla 2, así comolas diferencias de las medias de losfactoresentre los grupos de niños urbanos y semi-urbanos. Adicionalmente, se muestra que el tamaño de la diferencia entre las medias fue pequeño ( $d=<0.09$ ). De acuerdo a la Tabla 2, los factores de motivación (preferencias), habilidad (habilidades cognitivas) y de oportunidad ambiental (modelamiento y accesibilidad en la casa) mostraron una relación significativa con el C/N. En tanto, la percepción de barreras y accesibilidad en la escuela no revelaron una relación significativa con el CN.

\begin{tabular}{|c|c|c|}
\hline Frecuencia de consumo & $\begin{array}{l}\text { Niños Urbanos } \\
(n=800) \\
\%\end{array}$ & $\begin{array}{l}\text { Niños Semi-urbanos } \\
(n=634) \\
\%\end{array}$ \\
\hline Nunca & 5.3 & 5.2 \\
\hline Menos de 1 día a la semana & 11.5 & 9.5 \\
\hline Un día a la semana & 12.9 & 13.7 \\
\hline 2 - 4 días a la semana & 31.1 & 31.9 \\
\hline 5 - 6 días a la semana & 14.8 & 15.5 \\
\hline Todos los días, una vez al día & 14.9 & 11.7 \\
\hline Todos los días, dos veces al día & 4.3 & 6.6 \\
\hline Todos los días, más de dos veces al día & 5.4 & 6.0 \\
\hline
\end{tabular}


Tabla 2

Diferencias de medias respecto a los factores psicosociales, comparación de niños escolarizados urbanos y semi-urbanos

\begin{tabular}{|c|c|c|c|c|c|}
\hline \multicolumn{6}{|c|}{$\begin{array}{l}\text { Muestra total de seis ciudades del Estado de } \\
\text { Chihuahua, México }(n=1434)\end{array}$} \\
\hline & $\begin{array}{l}\text { Niños } \\
\text { Urbanos }\end{array}$ & $\begin{array}{c}\text { Niños } \\
\text { Semi- } \\
\text { Urbanos }\end{array}$ & & & \\
\hline & $(n=800)$ & $(n=634)$ & & & \\
\hline Factores & $M(D T)$ & $M(D T)$ & $t$ & $p$ & $\begin{array}{l}d \text { de } \\
\text { Cohen }\end{array}$ \\
\hline \multicolumn{6}{|l|}{ Motivación } \\
\hline Preferencias & $39.94(8.84)$ & $40.00(8.19)$ & -0.12 & 0.02 & 0.01 \\
\hline \multicolumn{6}{|l|}{ Habilidad } \\
\hline $\begin{array}{l}\text { Habilidades } \\
\text { cognitivas }\end{array}$ & $39.62(7.85)$ & $40.33(7.11)$ & -1.76 & 0.04 & 0.09 \\
\hline $\begin{array}{l}\text { Percepción de } \\
\text { barreras }\end{array}$ & $12.01(2.70)$ & $11.88(2.79)$ & 0.87 & 0.29 & $\mathrm{~N} / \mathrm{A}$ \\
\hline \multicolumn{6}{|l|}{$\begin{array}{l}\text { Oportunidad } \\
\text { ambiental }\end{array}$} \\
\hline Modelamiento & $14.42(3.34)$ & $14.70(3.13)$ & -1.65 & 0.05 & 0.09 \\
\hline $\begin{array}{l}\text { Accesibilidad } \\
\text { en la casa }\end{array}$ & $19.93(4.85)$ & $20.05(4.48)$ & -0.50 & 0.03 & 0.03 \\
\hline $\begin{array}{l}\text { Accesibilidad } \\
\text { en la escuela }\end{array}$ & $11.24(3.42)$ & $11.44(3.51)$ & -1.12 & 0.32 & $\mathrm{~N} / \mathrm{A}$ \\
\hline
\end{tabular}

\section{- CORRELACIONES ENTRE EL CONSUMO DE VERDURA Y LOS FACTORES DETERMINANTES}

Las correlaciones de Pearson entre el CN y sus determinantes resultaron ser significativas $(p<0.01)$ para ambos grupos de comparación (Tabla 3). Las correlaciones más altas en este estudio se obtuvieron en las habilidades cognitivas (rango: 0.51-0.63), en las preferencias (rango: $0.41-0.51$ ) y en la accesibilidad en la casa (rango: 0.42-0.51). Por otro lado, las correlaciones más bajas resultaron en la accesibilidad en la escuela (rango: 0.32-0.33) y en el modelamiento (rango: 0.30-0.35). Sin embargo, pese a que las correlaciones fueron de moderadas a buenas, las diferencias entre los índices de correlación de niños urbanos y semi-urbanos fueron mínimas. La prueba $Z$ mostró valores adecuados de significación para las preferencias $(p=0.02)$, habilidades cognitivas $(p=0.01)$, percepción de barreras ( $p$ $=0.04)$ y accesibilidad en la casa $(p=0.02)$.

Según la Tabla 3 la motivación (preferencias), habilidad (cognitivas) y oportunidad ambiental (accesibilidad en casa) mostraron las mejores correlaciones positivas ( $p \leq 0.04, r \geq 0.51$ ) y negativas (percepción de barreras; $r \leq-0.34$, $p=0.04)$ con el consumo de verdura. Los factores de oportunidad ambiental relacionados con el modelamiento y accesibilidad en la escuela no mostraron una correlación estadísticamente significativa con el consumo de verdura.

\section{- PREDICTORES DEL CONSUMO DE VERDURA EN NIÑOS ESCOLARES URBANOS Y SEMI- URBANOS}

Tras los análisis de correlación fueron seleccionados los predictores que obtuvieron correlaciones bivariadas superiores a 0.30 y con valores significativos en la prueba $Z$ (Tabla 3). De esta forma, según los valores beta estándar, las habilidades cognitivas ( $B=0.37)$, las preferencias o la accesibilidad en la casa (0.18) fueron mejores predictores que la percepción de barreras $(-0.05)$ en niños urbanos, mientras que este último factor $(-0.11)$

Tabla 3
Correlaciones de Pearson entre el consumo de verdura y los factores
determinantes en niños escolarizados urbanos y semi-urbanos

\begin{tabular}{|l|c|c|c|c|}
\hline & $\begin{array}{c}\text { Niños } \\
\text { Urbanos }\end{array}$ & $\begin{array}{c}\text { Niños Semi- } \\
\text { urbanos }\end{array}$ & \multicolumn{2}{|c|}{} \\
\cline { 2 - 4 } & $\begin{array}{c}\text { Consumo de } \\
\text { verdura }\end{array}$ & $\begin{array}{c}\text { Consumo de } \\
\text { verdura }\end{array}$ & \multicolumn{2}{|c|}{} \\
\hline Factores & Correlación $^{a}$ & Correlación $^{a}$ & $\boldsymbol{Z}$ & $\boldsymbol{P}$ \\
\hline
\end{tabular}

Motivación

Preferencias $\quad 0.51^{\star \star} \quad 0.41^{\star \star} \quad 2.31^{\text {** }} \quad 0.02^{\star \star}$

Habilidad

$\begin{array}{lllll}\begin{array}{l}\text { Habilidades } \\ \text { Cognitivas }\end{array} & 0.63^{* *} & 0.53^{\star *} & 2.83^{*} & 0.01^{*} \\ \begin{array}{l}\text { Percepción de } \\ \text { barreras }\end{array} & -0.43^{* *} & -0.34^{\star *} & -2.01^{\star *} & 0.04^{\star *}\end{array}$

Oportunidad

ambiental

$\begin{array}{lllll}\text { Modelamiento } & 0.35^{\star *} & 0.30^{\star *} & 1.01 & 0.31 \\ \begin{array}{l}\text { Accesibilidad } \\ \text { en la casa }\end{array} & 0.51^{\star *} & 0.42^{\star *} & 2.31^{\star *} & 0.02^{\star *} \\ \begin{array}{l}\text { Accesibilidad } \\ \text { en la escuela }\end{array} & 0.32^{\star *} & 0.33^{\star *} & 0.02 & 0.98\end{array}$

Nota: N/A: el dato no es aplicable.

a Significación estadística de las correlaciones ${ }^{* *} p<0.01$.

$Z=$ puntuación $Z$ de la comparación de las correlaciones entre niños escolares urbanos y semi-urbanos.

Significación estadística de las puntuaciones de la prueba de la $Z^{*} p<0.05$. $^{* *} p<0.01$ 
Tabla 4

Análisis de regresión de los predictores de motivación, habilidad y oportunidad ambiental del consumo de verdura en niños escolarizados urbanos y semi-urbanos

\begin{tabular}{|c|c|c|c|c|c|c|c|c|}
\hline & \multicolumn{4}{|c|}{ Niños escolares Urbanos $(n=800)$} & \multicolumn{4}{|c|}{ Niños escolares semi-urbanos $(n=634)$} \\
\hline & $\begin{array}{l}\text { Coef. B } \\
\text { no estand. }\end{array}$ & $\begin{array}{l}\text { Coef. } B \\
\text { estand. }\end{array}$ & $\begin{array}{l}\text { Correl. } \\
\text { semi-parcial }\end{array}$ & $p$ & $\begin{array}{l}\text { Coef. B } \\
\text { no estand. }\end{array}$ & $\begin{array}{l}\text { Coef. B } \\
\text { estand. }\end{array}$ & $\begin{array}{l}\text { Correl. } \\
\text { semi-parcial }\end{array}$ & $p$ \\
\hline & \multicolumn{4}{|c|}{$\begin{array}{c}\text { Modelo total }(F(4)=164.98 p<0.01) \\
\left.R^{2}=0.45 p<0.01\right)\end{array}$} & \multicolumn{4}{|c|}{$\begin{array}{c}\text { Modelo total }(F(4)=81.88) p<0.01) \\
\left.\qquad R^{2}=0.33 \quad p<0.01\right)\end{array}$} \\
\hline \multicolumn{9}{|l|}{ Motivación } \\
\hline Preferencias & 0.03 & 0.18 & 0.14 & 0.01 & 0.03 & 0.15 & 0.12 & 0.01 \\
\hline \multicolumn{9}{|l|}{ Habilidad } \\
\hline $\begin{array}{l}\text { Habilidades } \\
\text { cognitivas }\end{array}$ & 0.07 & 0.37 & 0.24 & 0.01 & 0.06 & 0.31 & 0.22 & 0.01 \\
\hline $\begin{array}{l}\text { Percepción } \\
\text { de barreras }\end{array}$ & -0.03 & -0.05 & -0.04 & 0.07 & -0.06 & -0.11 & -0.10 & 0.01 \\
\hline \multicolumn{9}{|l|}{$\begin{array}{l}\text { Oportunidad } \\
\text { ambiental }\end{array}$} \\
\hline $\begin{array}{l}\text { Accesibilidad } \\
\text { en la casa }\end{array}$ & 0.06 & 0.18 & 0.14 & 0.01 & 0.06 & 0.17 & 0.14 & 0.01 \\
\hline
\end{tabular}

tuvo más significación $(p \leq 0.07)$ en población semi-urbana. Por tanto, las preferencias, las habilidades cognitivas, la percepción de barreras y la accesibilidad se incluyeron en los modelos de análisis de regresión lineal múltiple. Sin embargo, el modelamiento y la accesibilidad en la escuela fueron eliminados de estos análisis.

En la Tabla 4 se detalla el análisis de regresión realizado para cada uno de los grupos estudiados. La varianza total explicada del CN obtenida en el grupo de niños urbanos fue del $45 \%$ y del $33 \%$ en niños semi-urbanos. Según los valores beta estándar, las habilidades cognitivas (0.37) las preferencias o accesibilidad en casa (0.18) fueron mejores predictores que la percepción de barreras (-0.05) en niños urbanos, mientras que este último factor tuvo más significación $(-0.11)$ en población semiurbana $(p \leq 0.07)$.

\section{DISCUSIÓN}

La teoría en la investigación es fundamental para aumentar la comprensión de los factores que predicen y explican el C FN (Guillaumie et al., 2010; Krølner et al., 2011; McClain et al., 2009; Rasmussen et al., 2006). Así, la teoría socio-cognitiva (Bandura, 1986), la teoría de la conducta planeada (Ajzen y Madden, 1986) y la teoría de la acción razonada (Ajzen y Fishbein, 1980) pueden facilitar la comprensión de la influencia de los factores sobre el C FN y por ende en el incremento de la eficiencia de las intervenciones. Además, la teoría permite el desarrollo de modelos teóricos y predictivos del C F/N (Brug, et al., 2006; Hardeman et al., 2005; Lehto et al., 2014; McClain et al., 2009).

Dada la importancia del uso de las teorías y el desarrollo de modelos, diversos estudios han analizado la predictibilidad de los factores psicosociales (Baranowski, Cullen y Baranowski, 1999; Guillaumie et al., 2010; Rasmussen et al., 2006), resaltando el valor de conocer la influencia de una o de múltiples teorías relacionada con la efectividad de las intervenciones en la promoción del C F/V (Diep et al., 2014). Sin embargo, algunos modelos con variables psicosociales derivadas de la teoría socio cognitiva (Bandura, 1986) y de la conducta planeada (Ajzen y Madden, 1986) han mostrado una baja predictibilidad en el C F/N (Baranowski et al., 1999; Guillaumie et al., 2010; Shaikh, Yaroch, Nebeling, Yeh y Resnicow, 2008). De esta forma, se ha recomendado el empleo de factores potentes que induzcan el cambio conductual y maximicen el efecto de la predictibilidad de las variables mediadoras (Brug et al., 2006; de Bruijn, Kroeze, Oenema y Brug, 2008; Gratton, Povey y Clark-Carter, 2007). No obstante, a partir la variabilidad reportada en el diseño de este tipo de estudios, se ha advertido la necesidad de la validación de las teorías y de los mediadores (Cerin, Barnett y Baranowski, 2009), a través de procedimientos sistemáticos y de diseños robustos (Cerin et al., 2009; Diep et 
al., 2014). Todo ello, para obtener una mayor efectividad en las intervenciones (Brug, Tak et al., 2008).

El abordaje del comportamiento dietético en modelos socio-ecológicos, generalmente se ha realizado sobre una conducta específica de consumo, a partir de tres niveles de influencia, mediante factores de motivación, habilidad y oportunidad ambiental (Rothschild, 1999; Sallis et al., 2008). De esta forma, en la exploración de las diferencias entre dichos niveles de influencia sobre el C F/V en niños escolares se ha encontraron que las preferencias constituyen la parte más importante de la motivación para el consumo y para el desarrollo de habilidades cognitivas en la toma de decisiones para un consumo saludable. Adicionalmente, la accesibilidad ha facilitado la disposición de oportunidades ambientales para el consumo diario.

En el presente estudio se halló que los niños escolarizados que residen en poblaciones urbanas y semi-urbanas poseen patrones semejantes de CN. Sin embargo, se encontraron pocas diferencias entre los predictores analizados en los grupos de comparación. El objetivo del presente estudio fue explorar el poder predictivo de factores psicosociales de motivación, habilidad y oportunidad ambiental asociados al $\mathrm{CN}$ en niños escolarizados de 10 a 12 años, que residen en poblaciones urbanas y semi-urbanas de seis ciudades del Estado de Chihuahua, México. Sin embargo, no se identificaron estudios previos sobre predictores del $\mathrm{C} / \mathrm{V}$ en niños escolarizados de poblaciones urbanas y semi-urbanas. Por ello, se hipotetizó que los niños residentes de poblaciones semi-urbanas mostrarían un menor gusto y menos preferencias por las verduras, menos habilidades cognitivas para el consumo diario, que percibirían mayores obstáculos para el consumo y menos accesibilidad a las mismas. Sin embargo, se invalida en parte esta hipótesis. Dado que, si bien es cierto que el $\mathrm{C} N$ resultó ser más o menos igual en ambas poblaciones (urbanas y semi-urbanas), los niños semi-urbanos reportaron mayor proporción de consumo diario de verduras, menos obstáculos para su consumo debido a que las barreras implican dificultades para comerlas, tardan más tiempo en comerlas y presentan dificultad para llevarlas a la escuela o debido a que se les antoja comer otro tipo de alimento.
Se ha hallado que el porcentaje de consumo diario proporcionalmente fue mayor en los niños semi-urbanos. También se comprobó que los factores de motivación (preferencias), de habilidad (habilidades cognitivas y percepción de barreras) y de oportunidad ambiental (accesibilidad en la casa) explicaron en población urbana y semi-urbana respectivamente del $33 \%$ al $45 \%$ de la varianza en el $\mathrm{C} / \mathrm{N}$, lo cual encaja con lo informado en estudios de revisión sobre modelos con variables psicosociales predictoras del C F/V. En dichos estudios se ha informado que la tendencia de predictibilidad ha sido menor al 30\% (Baranowski et al., 1999; De Bourdeaudhuii et al., 2006; Guillaumie et al., 2010; Shaikh et al., 2008). Sin embargo, Bere y Klepp $(2004,2005)$ han reportado varianzas superiores a las encontradas en el presente estudio. También se halló en ambos grupos del estudio, que las habilidades cognitivas mostraron una mayor capacidad predictiva respecto a las demás variables analizadas. En definitiva, los factores anteriormente mencionados facilitan la elección de alimentos saludables y predicen su consumo (Graham et al., 2013; Guillaumie et al., 2010; McClain et al., 2009; Moore et al., 2013; Rasmussen et al., 2006). Brug, Tak et al. (2008) encontraron que las preferencias ejercen una mayor influencia sobre el C F/V, además el apoyo parental activo y la accesibilidad son los mediadores ambientales de mayor potencia para el $C / N$ en niños escolarizados con peso normal y sobrepeso (De Bourdeaudhuii et al., 2006). En este estudio las preferencias, las habilidades cognitivas, la percepción de barreras y la accesibilidad en la casa han mostrado una capacidad predictiva del CN de acuerdo con las tendencias y estándares informados en estudios previos (Baranowski et al., 1999; De Bourdeaudhuii et al., 2006; Guillaumie et al., 2010; Shaikh et al., 2008; Wind et al., 2006). Asimismo, se halló que los factores de modelamiento y accesibilidad en la escuela no obtuvieron valores de predictibilidad significativos. Sin embargo, dichos factores han sido señalados como esenciales en las estrategias de intervención dirigidas a los padres, dado que ellos pueden influenciar el consumo de los niños y también en el llevar frutas y verduras a la escuela como una fuente de acceso a las mismas en el ámbito escolar (Bere y Klepp, 2004). En el contexto mexicano este acceso se ha impulsado 
a través de políticas gubernamentales que han jugado un papel estratégico mediante acuerdos nacionales que regulan la venta de $\mathrm{F} / \mathrm{V}$ en los centros escolares. Estas políticas han mejorado su accesibilidad, dado que su expendio representa el $11 \%$ del total de alimentos que se ofertan (Jiménez-Aguilar et al., 2014). Sin embargo, aún persiste el bajo consumo a nivel nacional y solo el $34 \%$ de los niños de edades entre 6 y 12 años cumple las recomendaciones de un consumo adecuado. Por ello, Jiménez-Aguilar et al. (2014) han concluido que los acuerdos para la salud alimentaria requieren para su implementación de política pública, de financiación y de una regulación eficiente. El presente estudio se ha realizado en niños del Estado de Chihuahua (México), dichos niños consumen menos F/V que otras regiones (centro y sur) del país (Encuesta Nacional de Salud y nutrición [ENSANUT], 2012). Por tanto, requieren de un fomento mayor para impulsar el consumo diario de $\mathrm{F} / \mathrm{V}$ al día. De acuerdo a Klepp et al.(2005) el C F/V puede depender de factores personales (preferencias, actitudes, conocimientos), socio-ambientales (familia, escuela) y físico-ambientales (políticas públicas, guías nutricionales y la accesibilidad). Por ello, nuestro trabajo exploró la capacidad predictiva de factores psicosociales asociados al $\mathrm{C} / \mathrm{V}$ tomando en cuenta el modelo sociológico de conducta saludable (Sallis et al., 2008) para explicar el C/ en niños escolarizados. Dichos factores que pueden emplearse en programas de intervención escolar bajo el marco de las políticas públicas dirigidas a regular la accesibilidad de manera eficaz en las escuelas centradas en el incremento de su consumo mediante factores psicosociales potentes (Pérez-Rodrigo et al., 2005). Además, los factores pueden contribuir a fomentar el consumo de otros alimentos y componentes nutricionales que mejoren la calidad de la dieta de los niños mexicanos como el consumo de vitaminas (Pedroza-Tobías et al., 2016).

Los resultados en el análisis multivariado de varianza mostraron puntuaciones medias significativas en los factores analizados. De esta forma, al estimar las diferencias de medias de los factores determinantes con la prueba $t$ entre los grupos de niños urbanos y semi-urbanos, se observó que solo las preferencias, las habilidades cognitivas, el modelamiento y la accesibilidad en la casa resultaron significativos.
No obstante, el tamaño de la diferencia entre dichas medias fue pequeño. Por otro lado, se compararon mediante la puntuación $Z$ las correlaciones de los factores con el CN, resultando significativas en ambos grupos de niños $(p<0.01)$. De esta forma, todos los factores que resultaron significativos fueron incluidos en los análisis de regresión, excepto el modelamiento y la accesibilidad en la escuela.

En los análisis de regresión se observaron pocas diferencias en la capacidad predictiva de los factores del CN entre los niños escolares urbanos y semi-urbanos. Cuatro de los seis predictores analizados mostraron una adecuada capacidad predictiva (preferencias, habilidades cognitivas, percepción de barreras y accesibilidad en la casa). Sin embargo, el factor de mayor consistencia fue el de habilidades cognitivas en ambos grupos (niños urbanos $B=<0.37$ y niños semi-urbanos $B=<0.31$ ). Las habilidades cognitivas generalmente se han identificado como una parte importante en la toma de decisiones para poder optar por alimentos saludables, dado que facilitan la efectividad de las estrategias de intervenciones nutricional es en las diversas poblaciones (Guillaumie et al., 2010; Mclain et al., 2009; Rasmussen et al., 2006). Estos resultados encajan con lo hallado en estudio previos sobre las preferencias como el determinante más importante en el incremento del C F/N y sobre la accesibilidad en casa como el moderador ambiental físico de mayor potencia en el CN (Brug, Tak etal., 2008). Sin embargo, en estudios desarrollados mediante metodologías y estrategias diferentes a los análisis de regresión, como lo es la modelización de ecuaciones estructurales, Neumark-Sztainer Wall, Perry y Story, (2003) obtuvieron una varianza en el C FN del 13\%, Sandvik et al. (2007) hallaron entre el $51 \%$ y el $62 \%$ de la varianza en la intención de comer fruta y del $27 \%$ al $38 \%$ de la varianza en el reporte del consumo actual de fruta. De la misma forma, Ochoa-Meza, Sierra, Pérez-Rodrigo, Aranceta Bartrina y Esparza-Del Villar (2015) encontraron una varianza del 48 $\%$ en el CN y una varianza total del $39 \%$ en las preferencias para comer fruta y su consumo actual. En definitiva, los estudios anteriormente mencionados han mostrado una tendencia entre baja y moderada en variables psicosociales predictoras del C F/N. Cabe mencionar, que en el presente estudio los niños semi-urbanos 
enfrentan con mejor eficiencia las barreras para el consumo de verduras y obtuvieron una mayor significatividad en la percepción de barreras ( $p=0.01)$ que los que residen en ciudades urbanas $(p=0.07)$.

Este estudio presenta algunas limitaciones. En primer lugar, el uso de un diseño de estudio transversal observacional para el análisis de los predictores, que no necesariamente implica la evaluación de la causalidad o de la capacidad predictiva de los factores (Larson, 1986). Para ello, tal vez hubiese sido más adecuado el empleo de estudios longitudinales. En segundo lugar, no se estimó el grado de influencia de la urbanización en el $\mathrm{C} / \mathrm{N}$, pues el tipo de población (urbana y semi-urbana) solo se utilizó para establecer diferencias entre los niños que residen en dichas poblaciones. Tercero, la generalización de los resultados no va más allá de la muestra utilizada, es decir, para una sola región del país. Además, tanto en la muestra total, como en las submuestras, no se consideraron las diferencias por sexo ni por nivel socioeconómico. Por ello, se sugiere que en futuras investigaciones se incluyan las diferentes regiones del país y sean analizadas mediante el diseño de estudios longitudinales y en muestras representativas nacionales. Adicionalmente, se deberán identificar y validar los predictores del C F/V, con la finalidad de incrementar la eficiencia de las intervenciones nutricionales dirigidas al colectivo de población, todo ello como una medida clave en la prevención de los principales problemas de salud pública como lo es la obesidad infantil en niños escolarizados (Aarestrup et al., 2014; Organización Panamericana de la Salud, OPS, 2007; World Health Organization [WHO], 2016). Asimismo, se sugiere la evaluación de las bases teóricas de los mediadores y la utilización de métodos de análisis de mediación, por ejemplo, la prueba de Sobel (MacKinnon, 2008; MacKinnon y Luecken, 2008) para estimar la significancia del efecto de la mediación en los programas de intervención (Cerin et al., 2009). También se propone el establecimiento de un control aleatorio de los grupos de estudio, tal y como lo realiza el consorcio Pro Children Project (PCHP) en la evaluación de los factores psicosociales del C F/V (Anderson, 2006). Asimismo, se sugiere analizar las variables mediadoras que son determinantes ambientales de la accesibilidad en la casa al C FN (Wyse,
Wolfenden y Bisquera, 2015) y como un elemento clave en los programas de intervención dirigido a los padres. Finalmente, se deberán evaluar los programas de intervención como lo propone el consorcio PCHP y evaluar el impacto de los efectos de los mediadores en el C F/N (Lehto et al., 2014; Tak, te Velde y Brug, 2008; Te Velde et al., 2008; Wind et al., 2006; Wind, te Velde, Brug, Sandvick y Kleep, 2010).

Una de las ventajas importantes en el presente estudio fue la utilización de una muestra representativa de los niños escolarizados de $5^{\circ}$ y $6^{\circ}$ grado, de ciudades urbanas y semi-urbanas del Estado de Chihuahua (México) y la utilización de un cuestionario válido y fiable para medir factores determinantes de motivación, habilidad y oportunidad ambiental asociados al CN, con el cual se obtuvo un adecuado porcentaje de respuesta en la aplicación del mismo. En conclusión, el presente estudio propone que el diseño de estrategias de intervención dirigidas a fomentar el consumo de verduras en el contexto escolar mexicano se dirija a incrementar las preferencias y las habilidades cognitivas para el consumo diario de verduras, así como disminuir la percepción de barreras y facilitar la accesibilidad a las verduras en la casa para el logro de intervenciones efectivas.

\section{- Conflicto de intereses}

Los autores declaran no tener ningún conflicto de intereses.

\section{- Agradecimientos}

Los autores agradecen la colaboración de los niños, personal docente y administrativo de las escuelas que participaron activamente en este estudio. La presente investigación fue aprobada por el Comité de Bioética de la Universidad Autónoma de Ciudad Juárez, Chihuahua (México) y se realizó sin recibir financiación alguno de agencias públicas o privadas, nacionales o internacionales.

\section{REFERENCIAS}

Aarestrup, A. K., Krølner, R., Jørgensen, T. S., Evans, A., Due, P., y Tjørnhøj-Thomsen, T. (2014). Implementing a free school-based fruit and vegetable programme: Barriers and facilitators experienced by pupils, teachers and produce suppliers in the Boost study. BMC Public Health, 14, 146. doi: 10.1186/1471-2458-14-146

Agudo, A. (2005). Measuring intake of fruit and vegetables. Background paper for the joint FAO/WOH Workshop on fruit and Vegetable for health. Recuperado de http://apps.who.int/iris/ 
bitstream/10665/43144/1/9241592826 eng. pdf? $v a=1$

Ajzen, I., y Fishbein, M. (1980). Understanding attitudes and predicting social behaviour. Englewood Cliffs, NJ: Prentice-Hall.

Ajzen, I., y Madden, T. J. (1986). Prediction of goaldirected behavior: Attitudes, intentions, and perceived behavioral control. Journal of Experimental Social Psychology, 22, 453-474. doi: 10.1016/00221031 (86)90045-4

Anderson, A. S. (2006). The Pro-children Project-a crossnational approach to increasing fruits and vegetables in the next generation and onwards. International Journal of Behavioral Nutrition and Physical Activity, 3, 26. doi: 10.1186/1479-5868-3-26

Bandura, A. (1986). Social foundations of thought and action: A social cognitive theory. Englewood Cliffs, NJ: Prentice-Hall.

Baranowski, T. (2011). Understanding the behavioral linkages needed for designing effective intervention to increase fruit and vegetables intake diverse population. Journal of the American Dietetic Association, 117 , 1472-1475. doi: 10.1016/i.jada.2011.07.014

Baranowski, T., Cullen, K. W., y Baranowski, J. (1999). Psychosocial correlates of dietary intake: Advancing dietary intervention. Annual Review of Nutrition, 19, 17-40. doi: 10.1146/annurev.nutr.19.1.17

Baranowski, T., Diep, C., y Baranowski, J. (2013). Influences on children's dietary behavior, and innovative attempts to change it. Annals of Nutrition and Metabolism, 62 (Suppl. 3), 38-46. doi: $10.1159 / 000351539$

Bere, E., y Klepp, K. I. (2004). Correlates of fruit and vegetable intake among Norwegian schoolchildren: parental and self-reports. Public Health Nutrition, 7, 991-998. doi: 10.1079/PHN2004619

Bere, E., y Klepp, K. I. (2005). Changes in accessibility and preferences predict children's future fruit and vegetable intake. International Journal Behavioral Nutrition and Physical Activity, 2, 15. doi: 10.1186/1479-5868-2-15

Blanchard, C. M., McGannon, K. R., Spence, J. C., Rhodes, R. E., Nehl, E., Baker, F., y Bostwick, J. (2005). Social ecological correlates of physical activity in normal weight, overweight, and obese individuals. International Journal of Obesity, 29, 720-726. doi: 10.1038/si.ijo.0802927

Boeing, H., Bechthold, A., Bub, A., Ellinger, S., Haller, D., Kroke, A.,... Watzl, B. (2012). Critical review: vegetables and fruit in the prevention of chronic diseases. European Journal of Nutrition, 51, 637-663. doi: 10.1007/s00394-012-0380-y
Brug, J., de Vet, E., de Nooijer, J., y Verplanken, B. (2006). Predicting fruit consumption: Cognitions, intention, and habits. Journal of Nutrition Education and Behavior, 38, 73-81. doi: 10.1016/i.ineb.2005.11.027

Brug, J., Kremers, S. P., Lenthe, F. V., Ball, K., y Crawford, D. (2008). Environmental determinants of healthy eating: in need of theory and evidence. Proceedings of the Nutrition Society, 67, 307-316. doi: 10.1017/ $\underline{\mathrm{s} 0029665108008616}$

Brug, J., Tak, N. I., Velde, S. J., Bere, E., y De Bourdeaudhuii, I. (2008). Taste preferences, liking and other factors related to fruit and vegetable intakes among schoolchildren: Results from observational studies. British Journal of Nutrition, 99, 7-14. doi: 10.1017/S0007114508892458

Cardon, G. M., Van Acker, R., Seghers, J., De Martelaer, K., Haerens, L. L., y De Bourdeaudhuii, I. M. (2012). Physical activity promotion in schools: Which strategies do schools (not) implement and which socioecological factors are associated with implementation? Health Education Research, 27, 470-483. doi: 10.1093/her/ cys043

Cerin, E., Barnett, A., y Baranowski, T. (2009). Testing theories of dietary behavior change in youth using the mediating variable model with intervention programs. Journal of Nutrition Education and Behavior, 41, 309. 318. doi: 10.1016/i.jneb.2009.03.129

Cohen, J. (1992). A power primer. Psychological Bulletin, 172, 155.

De Bourdeaudhuii, I., Klepp, K. I., Due, P., Rodrigo, C. P., De Almeida, M. D. V., Wind, M.,... Brug, J. (2005). Reliability and validity of a questionnaire to measure personal, social and environmental correlates of fruit and vegetable intake in 10-11-year-old children in five European countries. Public Health Nutrition, 8, 189200. doi: 10.1079/PHN2004673

De Bourdeaudhuii, I., Yngve, A., Te Velde, S. J., Klepp, K. I., Rasmussen, M., Thorsdottir, I., ... Brug, J. (2006). Personal, social and environmental correlates of vegetable intake in normal weight and overweight 9 to 13-year old boys. International Journal of Behavioral Nutrition and Physical Activity, 3, 37. doi: 10.1186/1479-5868-3-37

De Bruiin, G. J., Kroeze, W., Oenema, A., y Brug, J. (2008). Saturated fat consumption and the theory of planned behaviour: exploring additive and interactive effects of habit strength. Appetite, 51, 318-323. doi: 10.1016/i.appet.2008.03.012

D'Haese, S., Vanwolleghem, G., Hinckson, E., De Bourdeaudhuii, I., Deforche, B., Van Dyck, D., y Cardon, G. (2015). Cross-continental comparison of the association between the physical environment and 
active transportation in children: A systematic review. International Journal of Behavioral Nutrition and Physical Activity, 12, 145-158. doi: 10.1186/s12966$\underline{015-0308-z}$

Diep, C. S., Chen, T. A., Davies, V. F., Baranowski, J. C., y Baranowski, T. (2014). Influence of behavioral theory on fruit and vegetable intervention effectiveness among children: A meta-analysis. Journal of Nutrition Education and Behavior, 46, 506-546. doi: 10.1016/i. ineb.2014.05.012

Encuesta Nacional de Salud y Nutrición. ENSANUT (2012). Resultados nacionales, México. Síntesis ejecutiva. Recuperado de http://ensanut.insp.mx/ resultados principales.php\#.VEIFBxznSSg

Flay, B. R., y Petraitis, J. (1994). A new theory of health behavior with implications for preventive interventions. Advances in Medical Sociology, 4, 19-44.

Gentile, D. A., Welk, G., Eisenmann, J. C., Reimer, R. A., Walsh, D. A., Russell, D. W.,... Fritz, K. (2009). Evaluation of a multiple ecological level child obesity prevention program: Switch ${ }^{\circledR}$ what you Do, View, and Chew. BMC Medicine, 7, 49-60. doi: 10.1186/17417015-7-49

Graham, D. J., Pelletier, J. E., Neumark-Sztainer, D., Lust, K., y Laska, M. N. (2013). Perceived social-ecological factors associated with fruit and vegetable purchasing, preparation, and consumption among young adults. Journal of the Academy of Nutrition and Dietetics, 113 , 1366-1374. doi: 10.1016/i.jand.2013.06.348

Gratton, L., Povey, R., y Clark-Carter, D. (2007). Promoting children's fruit and vegetable consumption: Interventions using the Theory of Planned Behaviour as a framework. British Journal of Health Psychology, 12, 639-650. doi: 10.1348/135910706X171504

Guillaumie, L., Godin, G., y Vézina-Im, L. A. (2010). Psychosocial determinants of fruit and vegetable intake in adult population: A systematic review. International Journal of Behavioral Nutrition and Physical Activity, 7, 12. doi: 10.1186/1479-5868-7-12

Hardeman, W., Sutton, S., Griffin, S., Johnston, M., White, A., Wareham, N. J., y Kinmonth, A. L. (2005). A causal modelling approach to the development of theory-based behaviour change programmes for trial evaluation. Health Education Research, 20, 676-687. doi: 10.1093/her/cyh022

Instituto Nacional de Estadística, Geografía e Informática. (2010). Censo de población y vivienda. resultados definitivos, tabulados básicos. México: INEGI. Recuperado de www.inegi.org.mx

Jiménez-Aguilar, A., Gaona-Pineda, E. B., Mejía Rodríguez, F., Gómez-Acosta, L. M., Méndez-Gómez Humarán, I., y Flores-Aldana, M. (2014). Consumption of fruits and vegetables and health status of Mexican children from the National Health and Nutrition Survey 2012. Salud Pública de México, 56(Suppl. 2), 103-112.

Klepp, K.I., Pérez-Rodrigo, C., De Bourdeaudhuijc, I., Due, P., Elmadfa, I., Haraldsdóttirf, P.,... Brug, J. (2005). Promoting fruit and vegetable consumption among European schoolchildren: Rationale, conceptualization and design of the pro children project. Annals of Nutrition and Metabolism, 49, 212-220. doi: $10.1159 / 000087245$

Krølner, R., Jørgensen, T. S., Aarestrup, A. K., Christiansen, A. H., Christensen, A. M., y Due, P. (2012). The Boost study: design of a school-and communitybased randomised trial to promote fruit and vegetable consumption among teenagers. BMC Public Health, 12, 191. doi: 10.1186/1471-2458-12-191

Krølner, R., Rasmussen, M., Brug, J., Klepp, K. I., Wind, M., y Due, P. (2011). Determinants of fruit and vegetable consumption among children and adolescents: A review of the literature. Part II: Qualitative studies. International Journal of Behavioral Nutrition and Physical Activity, 8, 1-38. doi: 10.1186/1479-5868-8-112

Larson, E. (1986). Evaluating validity of screening tests. Nursing Research, 35, 186-187.

Lehto, E., Ray, C., Te Velde, S., Petrova, S., Duleva, V., Krawinkel, M.,... Yngve, A. (2014). Mediation of parental educational level on fruit and vegetable intake among schoolchildren in ten European countries. Public Health Nutrition, 18, 89-99. doi: 10.1017/ S136898001300339X

MacKinnon, D. P. (2008). Introduction to statistical mediation analysis. Mahwah, NJ: LEA.

MacKinnon, D. P., y Luecken, L. J. (2008). How and for whom? Mediation and moderation in health psychology. Health Psychology, 27, S99-S100. doi: 10.1037/0278-6133.27.2(Suppl.).S99

McClain, A. D., Chappuis, C., Nguyen-Rodriguez, S. T., Yaroch, A. L., y Spruijt-Metz, D. (2009). International Journal of Behavioral Nutrition and Physical Activity. International Journal of Behavioral Nutrition and Physical Activity, 6, 54. doi: 10.1 186/1479-5868-6-54 Moore, L., de Silva-Sanigorski, A., y Moore, S. N. (2013).

A socio-ecological perspective on behavioural interventions to influence food choice in schools: Alternative, complementary or synergistic? Public Health Nutrition, 16, 1000-1005. doi: 10.1017/ S1368980012005605

Neumark-Sztainer, D., Wall, M., Perry, C., y Story, M. (2003). Correlates of fruit and vegetable intake among adolescents. Findings from Proyect EAT. Preventive Medicine, 37, 198-208. doi: 10.1016/S00917435(03)001 14-2 
Ochoa-Meza, G., Sierra, J.C., Pérez-Rodrigo, C., y Aranceta-Bartrina, J. (2014). Validación del cuestionario Pro Children Project para evaluar factores psicosociales del consumo de fruta y verdura en México. Salud Pública de México. 56, 65-179.

Ochoa-Meza, G., Sierra, J. C., Pérez-Rodrigo, C., Aranceta Bartrina, J., y Esparza-Del Villar, Ó. A. (2014). Fiabilidad y validez de la versión mexicana del cuestionario Pro Children Project. Nutrición Hospitalaria, 30, 293-300. doi: 10.3305/nh.2014.30.2.7595

Ochoa-Meza, G., Sierra, J. C., Pérez-Rodrigo, C., Aranceta Bartrina, J., y Esparza-Del Villar, Ó. A. (2015). Validez e invarianza factorial de un modelo socio-ecológico para explicar el consumo de fruta en niños escolares mexicanos. Nutrición Hospitalaria, 31 , 649-657. doi: 10.3305/nh.2015.31.2.8317

Organización Panamericana de la Salud [OPS]. (2007). Estrategia regional y plan de acción para un enfoque integrado sobre la prevención y el control de las enfermedades crónicas. Washington, D.C.: OPS.

Pedroza-Tobías, A., Hernández-Barrera, L., López-Olmedo, N., García-Guerra, A., Rodríguez-Ramírez, S., RamírezSilva, I., ...Rivera, J. A. (2016). Usual vitamin intakes by Mexican populations. The Journal of Nutrition, 146, 1866S-1873S. doi: 10.3945/in.115.219162

Pérez-Rodrigo, C., Wind, M., Hildonen, C., Bjelland, M., Aranceta, J., Klepp, K. I., y Brug, J. (2005). The pro children intervention: Applying the intervention mapping protocol to develop a school-based fruit and vegetable promotion programme. Annals of Nutrition and Metabolism, 49, 267-277. doi: $10.1159 / 000087249$

Rasmussen, M., Krolner, R., Klepp, I. K., Lytle, L., Brug, J., Bere, E., y Due, P. (2006). Determinants Fruit and Vegetable among Children and Adolescents: Review literature. Part I: Quantitative studies. International Journal of Behavioral Nutrition and Physical Activity, 3, 22-41. doi: 10.1186/1479-5868-3-22

Robinson, T. (2008). Applying the socio-ecological model to improving fruit and vegetable intake among low-income African Americans. Journal Community Health 33, 395-406. doi: 10.1007/s10900-0089109-5

Rothschild, M. L. (1999). Carrots, sticks, and promises: A conceptual framework for the management of public health and the social issue behaviors. The Journal of Marketing, 63, 24-37.

Sallis, J. F., Owen, N., y Fisher, E. B. (2008). Ecological models of health behavior. En K. Glanz, B. K. Rimer, y K. Viswanath (Eds.), Health behavior and health education: Theory, research, and practice (pp. 465486). San Francisco, CA: Jossey Bass.
Sandvik, C., Gjestad, R., Brug, J., Rasmussen, M., Wind, M., Wolf, A., ... Klepp, K. I. (2007). The application of a social cognition model in explaining fruit intake in Austrian, Norwegian and Spanish schoolchildren using structural equation modelling. International Journal of Behavioral Nutrition and Physical Activity, 4, 57. doi: 10.1186/1479-5868-4-57

Servicios Educativos del Estado de Chihuahua. (2014). Departamento de estadística. México: Servicios Educativos del Estado de Chihuahua. Recuperado de http://seech.gob.mx/estadistica/paginas asp/ inicio2009.asp

Shaikh, A. R., Yaroch, A. L., Nebeling, L., Yeh, M. C., y Resnicow, K. (2008). Psychosocial predictors of fruit and vegetable consumption in adults: A review of the literature. American Journal of Preventive Medicine, 34, 535-543. doi: 10.1016/i.amepre.2007.12.028 SPSS. (2011). Statistical Package for Social Sciences for Windows version 20.0. Chicago, IL: SPSS Inc.

Tak, N. I., te Velde, S. J., y Brug, J. (2008). Are positive changes in potential determinants associated with increased fruit and vegetable intakes among primary schoolchildren? Results of two intervention studies in the Netherlands: The Schoolgruiten Project and the Pro Children Study. International Journal of Behavioral Nutrition and Physical Activity, 5, 21 . doi: 10.1186/1479-5868-5-21

Te Velde, S. J., Brug, J., Wind, M., Hildonen, C., Bjelland, M., Perez-Rodrigo, C., y Klepp, K. I. (2008). Effects of a comprehensive fruit-and vegetable-promoting school-based intervention in three European countries: The Pro Children Study. British Journal of Nutrition, 99, 893-903. doi: http://dx.doi. org/10.1017/s000711450782513X

Townsend, N., y Foster, C. (2011). Developing and applying a socio-ecological model to the promotion of healthy eating in the school. Public Health Nutrition, 16, 1101-1108. doi: 10.1017/ S1368980011002655

Vanwolleghem, G., Schipperijn, J., Gheysen, F., Cardon, G., De Bourdeaudhuij, I., y Van Dyck, D. (2016). Children's GPS-determined versus self-reported transport in leisure time and associations with parental perceptions of the neighborhood environment. International Journal of Health Geographics, 15, 16. doi: 10.1371/journal.pone.0156531

Wind, M., De Bourdeaudhuij, I., te Velde, S. J., Sandvik, C., Due, P., Klepp, K. I., y Brug, J. (2006). Correlates of fruit and vegetable consumption among 11 -yearold Belgian-Flemish and Dutch schoolchildren. Journal of Nutrition Education and Behavior, 38, 211-221. doi: 10.1016/i.jneb.2006.02.011 
Wind, M., te Velde, S. J., Brug, J., Sandvik, C., y Klepp, K. I. (2010). Direct and indirect association between environmental factors and fruit intake, mediation by psychosocial factors: The Pro Children study. Public Health Nutrition, 13, 1736-1745. doi: 10.1017/ S1368980010002302

Wolf, A., Yngve, A., Elmadfa, I., Poortvliet, E., Ehren blad, B., Pérez-Rodrigo, C.,... Klepp, K. I. (2005). Fruit and vegetable intake of mothers of 11 -year-old children in nine European countries: The Pro Children Crosssectional Survey. Annals of Nutrition and Metabolism, 49, 246-254. doi: 10.1159/000087248

World Health Organization (WHO). (2016). Implementation plan for the recommendations of the Commission on Ending Childhood Obesity. Recuperado de http://www. who.int/end-childhood-obesity/en/
Wyse, R., Wolfenden, L., y Bisquera, A. (2015). Characteristics of the home food environment that mediate immediate and sustained increases in child fruit and vegetable consumption: mediation analysis from the Healthy Habits cluster randomised controlled trial. International Journal ofBehaviora/Nutrition and Physical Activity, 12, 118. doi: 10.1186/s12966-015-0281-6 Yngve, A., Wolf, A., Poortvliet, E., Elmadfa, I., Brug, J., Ehrenblad, B.,... Klepp, K. I. (2005). Fruit and vegetable intake in a sample of 11 -year-old children in 9 European countries: The pro children cross-sectional survey. Annals of Nutrition and Metabolism, 49, 236 245. doi: $10.1159 / 000087247$ 